\title{
RECURSIVE DETERMINATION OF THE SUM-OF-DIVISORS FUNCTION
}

JOHN A. EWELL

\begin{abstract}
A recursive scheme for determination of the sum-of-divisors function is presented. As all of the formulas involve triangular numbers, the scheme is therefore compared for efficiency with another known recursive triangular-number formula for this function.
\end{abstract}

For each positive integer $n, \sigma(n)$ denotes the sum of the positive divisors of $n$. Recently Ewell [1] derived two recursive schemes for computing the values $\sigma(n)$, and regarding efficiency of computation compared each of them with the well-known pentagonal-number formula of Euler. In the present discussion we derive another recursive scheme and show that among the four known recursive determinations of $\sigma$ this one ranks no worse than second with regard to efficiency. We prepare the way for an easy statement of our result by defining an auxiliary function $\alpha$ as follows. For each positive integer $n$, we express $n$ uniquely as $n=2^{b(n)} O(n)$ where $b(n)$ is a nonnegative integer and $O(n)$ is odd. Now define $\alpha(n)=\left(2^{b(n)+1}-3\right) \sigma(O(n))$.

THEOREM. For each positive integer $m$,

$$
\begin{aligned}
& \sigma(2 m-1)-\sum_{k=0}\{\alpha(2 m-1-(2 k+1)(4 k+1)) \\
&+\alpha(2 m-1-(2 k+1)(4 k+3))\} \\
&++\sigma(2 m-1-(2 k+2)(4 k+5))\} \\
&+\sum_{k=0}\{\sigma(2 m-1-(2 k+2)(4 k+3)) \\
&= \begin{cases}n(n+1) / 2, & \text { if } 2 m-1=n(n+1) / 2, \\
0, & \text { otherwise, }\end{cases}
\end{aligned}
$$

Received by the editors February 6, 1978.

AMS (MOS) subject classifications (1970). Primary 10A20; Secondary 10A35.

Key words and phrases. Recurrences, sum of the positive divisors of a positive integer, identities. (c) 1979 American Mathematical Society $0002-9939 / 79 / 0000-0056 / \$ 02.00$ 
and

$$
\begin{aligned}
\alpha(2 m) & -\sum_{k=0}\{\sigma(2 m-(2 k+1)(4 k+1))+\sigma(2 m-(2 k+1)(4 k+3))\} \\
& +\sum_{k=0}\{\alpha(2 m-(2 k+2)(4 k+3))+\alpha(2 m-(2 k+2)(4 k+5))\} \\
= & \begin{cases}-n(n+1) / 2, & \text { if } 2 m=n(n+1) / 2, \\
0, & \text { otherwise, }\end{cases}
\end{aligned}
$$

where in both (1) and (2) summation extends as far as the arguments are positive.

Proof. Our proof depends on the following two identities due respectively to Euler and Gauss.

$$
\begin{gathered}
\prod_{n=1}^{\infty}\left(1+x^{n}\right)\left(1-x^{2 n-1}\right)=1 \\
\prod_{n=1}^{\infty}\left(1-x^{2 n}\right)\left(1+x^{n}\right)=\sum_{n=0}^{\infty} x^{n(n+1) / 2}
\end{gathered}
$$

For proofs see [2, pp. 277-284]. Since $1-x^{2 n}=\left(1-x^{n}\right)\left(1+x^{n}\right)$, we use Euler's identity to express Gauss's identity in the equivalent form

$$
\prod_{n=1}^{\infty}\left(1-x^{n}\right) \prod_{n=1}^{\infty}\left(1-x^{2 n-1}\right)^{-2}=\sum_{n=0}^{\infty} x^{n(n+1) / 2}
$$

Briefly, set $F(x)=\sum x^{n(n+1) / 2}$. Now take the logarithmic derivative of the foregoing identity and multiply the resulting identity by $x$ to obtain

$$
\sum_{n=1}^{\infty} \frac{n x^{n}}{1-x^{n}}-2 \sum_{n=1}^{\infty} \frac{(2 n-1) x^{2 n-1}}{1-x^{2 n-1}}=-\frac{x F^{\prime}(x)}{F(x)}
$$

It is well known that the first series on the left of (3), a "Lambert" series, generates $\sigma(n)$ : i.e.,

$$
\sum_{n=1}^{\infty} \frac{n x^{n}}{1-x^{n}}=\sum_{n=1}^{\infty} \sigma(n) x^{n} .
$$

The second series is perhaps less well known, but straightforward algebraic manipulation shows that

$$
\begin{aligned}
\sum_{n=1}^{\infty} \frac{(2 n-1) x^{2 n-1}}{1-x^{2 n-1}} & =\sum_{n=1}^{\infty} x^{n} \sum_{\substack{d \mid n \\
d \text { odd }}} d \\
& =\sum_{m=1}^{\infty} \sigma(2 m-1) x^{2 m-1}+\sum_{m=1}^{\infty} \sigma(O(2 m)) x^{2 m}
\end{aligned}
$$

Thus, identity (3) becomes

$$
\sum_{m=1}^{\infty} \sigma(2 m-1) x^{2 m-1}-\sum_{m=1}^{\infty} \alpha(2 m) x^{2 m}=x F^{\prime}(x) / F(x) .
$$


Now, separating the even and odd triangular numbers $n(n+1) / 2$ by the least positive residues of $n(\bmod 4)$, we write

$$
\begin{aligned}
& \left\{\sum_{m=1}^{\infty} \sigma(2 m-1) x^{2 m-1}\right\} F(x) \\
& =\sum_{m=1}^{\infty} x^{2 m-1}\left\{\sigma(2 m-1)+\sum_{k=0}[\sigma(2 m-1-(2 k+2)(4 k+3))\right. \\
& +\sigma(2 m-1-(2 k+2)(4 k+5))]\} \\
& +\sum_{m=1}^{\infty} x^{2 m}\left\{\sum_{k=0}[\sigma(2 m-(2 k+1)(4 k+1))\right. \\
& +\sigma(2 m-(2 k+1)(4 k+3))]\}
\end{aligned}
$$

and,

$$
\begin{aligned}
& \left\{\sum_{m=1}^{\infty} \alpha(2 m) x^{2 m}\right\} F(x) \\
& =\sum_{m=1}^{\infty} x^{2 m-1}\left\{\sum_{k=0}[\alpha(2 m-1-(2 k+1)(4 k+1))\right. \\
& +\alpha(2 m-1-(2 k+1)(4 k+3))]\} \\
& +\sum_{m=1}^{\infty} x^{2 m}\left\{\alpha(2 m)+\sum_{k=0}[\alpha(2 m-(2 k+2)(4 k+3))\right. \\
& +\alpha(2 m-(2 k+2)(4 k+5))]\} .
\end{aligned}
$$

We then substitute these last two developments into the identity

$$
\begin{gathered}
\left\{\sum_{m=1}^{\infty} \sigma(2 m-1) x^{2 m-1}\right\} F(x)-\left\{\sum_{m=1}^{\infty} \alpha(2 m) x^{2 m}\right\} F(x) \\
=x F^{\prime}(x)=\sum_{n=1}^{\infty} \frac{n(n+1)}{2} x^{n(n+1) / 2}
\end{gathered}
$$

equate coefficients of odd powers $x^{2 m-1}$ to obtain recurrence (1) and equate coefficients of even powers $x^{2 m}$ to obtain recurrence (2).

REMARKS. One of the three recurrences discussed in [1] is

$$
\begin{aligned}
& \sum_{k=0}(-1)^{k}(2 k+1) \sigma(n-k(k+1) / 2) \\
& \quad= \begin{cases}(-1)^{j+1} j(j+1)(2 j+1) / 6, & \text { if } n=j(j+1) / 2, \\
0, & \text { otherwise. }\end{cases}
\end{aligned}
$$


Like our recurrences (1) and (2) this recurrence involves triangular numbers. Since $\sigma$ is multiplicative and therefore $\sigma(n)=\left(2^{b(n)+1}-1\right) \sigma(O(n))$, we suppose that we are given a large odd number $n$ and investigate efficiency of computation of $\sigma(n)$ by recurrences (1) and (4). Theoretically we deduce that each of the recurrences needs about $\sqrt{2 n}$ of the values, $\sigma(j), 1<j<n$. But, practically, let us take a not-too-large value of $n$, say $n=63$, partially compute $\sigma(63)$ by each of the recurrences and possibly observe some noteworthy differences.

By recurrence (1),

$$
\begin{aligned}
\sigma(63)= & \left(2^{2}-3\right) \sigma(31)+\left(2^{5}-3\right) \sigma(3)+\left(2^{2}-3\right) \sigma(9)+\left(2^{3}-3\right) \sigma(15) \\
& +\left(2^{2}-3\right) \sigma(21)+\left(2^{4}-3\right) \sigma(1)-\sigma(57)-\sigma(35)-\sigma(53)-\sigma(27) .
\end{aligned}
$$

By recurrence (4),

$$
\begin{aligned}
\sigma(63)= & 3 \sigma(62)-5 \sigma(60)+7 \sigma(57)-9 \sigma(53)+11 \sigma(48)-13 \sigma(42) \\
& +15 \sigma(35)-17 \sigma(27)+19 \sigma(18)-21 \sigma(8) .
\end{aligned}
$$

Although each recurrence uses 10 lower values, we observe that recurrence (1) uses smaller values $\sigma(j)$ by separating the binary and odd parts of $j$. (This is something that any high-speed computing machine can do easily.) Also, recurrence (1) avoids coefficients such as the $2 k+1$ of (4).

\section{REFERENCES}

1. J. A. Ewell, Recurrences for the sum of divisors, Proc. Amer. Math. Soc. 64 (1977), 214-218.

2. G. H. Hardy and E. M. Wright, An introduction to the theory of numbers, 4th ed., Clarendon Press, Oxford, 1960. 60115

Department of Mathematical Sciences, Northern Illinois University, DeKalb, Illinois 